\title{
Utility of Elastography with Endoscopic Ultrasonography Shear-Wave Measurement for Diagnosing Chronic Pancreatitis
}

\author{
Yasunobu Yamashita ${ }^{1}$, Kensuke Tanioka $^{2}$, Yuki Kawaji ${ }^{1}$, Takashi Tamura ${ }^{1}$, Junya Nuta ${ }^{1}$, Keiichi Hatamaru ${ }^{1}$, Masahiro \\ Itonaga $^{1}$, Takeichi Yoshida ${ }^{1}$, Yoshiyuki Ida ${ }^{1}$, Takao Maekita ${ }^{1}$, Mikitaka Iguchi ${ }^{1}$, and Masayuki Kitano ${ }^{1}$ \\ ${ }^{1}$ Second Department of Internal Medicine, Wakayama Medical University, and ${ }^{2}$ Clinical Study Support Center, Wakayama Medical University \\ Hospital, Wakayama, Japan
}

Background/Aims: Rosemont classification (RC) with endoscopic ultrasonography (EUS) is important for diagnosing chronic pancreatitis (CP) but is based only on subjective judgement. EUS shear wave measurement (EUS-SWM) is a precise modality based on objective judgment, but its usefulness has not been extensively studied yet. This study evaluated the utility of EUS-SWM for diagnosing CP and estimating $\mathrm{CP}$ severity by determining the presence of endocrine dysfunction along with diabetes mellitus (DM). Methods: Between June 2018 and December 2018, 52 patients who underwent EUS and EUS-SWM were classified into two groups according to RC: non-CP (indeterminate $\mathrm{CP}$ and normal) and CP (consistent and suggestive of CP). The EUSSWM value by shear wave velocity was evaluated with a median value. The EUS-SWM value was compared with RC and the number of EUS features. The diagnostic accuracy and cutoff value of EUS-SWM for CP and DM and its sensitivity and specificity were calculated. Results: The EUS-SWM value significantly positively correlated with the RC and the number of EUS features. The EUS-SWM values that were consistent and suggestive of $\mathrm{CP}$ were significantly higher than that of normal. The area under the receiver operating characteristic (AUROC) curve for the diagnostic accuracy of EUS-SWM for CP was 0.97 . The cutoff value of 2.19 had $100 \%$ sensitivity and $94 \%$ specificity. For endocrine dysfunction in CP, the AUROC was 0.75 . The cutoff value of 2.78 had $70 \%$ sensitivity and 56\% specificity. Conclusions: EUS-SWM provides an objective assessment and can be an alternative diagnostic tool for diagnosing CP. EUS-SWM may also be useful for predicting the presence of endocrine dysfunction. (Gut Liver 2020;14:659-664)
Key Words: Pancreatitis, chronic; Shear wave EUS elastography; Rosemont classification; Diabetes mellitus

\section{INTRODUCTION}

The estimated annual prevalence and incidence of chronic pancreatitis (CP) were 52.4/100,000 and 14.0/100,000, respectively. The sex ratio (male/female) of patients was reported to be 4.6 and patients' mean age was 62.3 years. ${ }^{1} \mathrm{CP}$ is the inflammation of the pancreas, which leads to permanent impairment of exocrine and endocrine functions. Therefore, it is currently considered as an important healthcare problem. However, there is no consensus regarding the severity grading of $\mathrm{CP}$ in histology. ${ }^{2}$ Moreover, Dewitt et al. ${ }^{3}$ reported endoscopic ultrasonography (EUS)-guided trucut biopsy is not enough for evaluating the grading of $\mathrm{CP}$.

EUS is thought to be one of the most reliable and efficient diagnostic modalities for pancreatobiliary diseases because of its superiority to any other modalities with respect to spatial resolution. ${ }^{4.5}$ Nowadays, EUS can be considered as one of the modalities for the morphological diagnosis of $\mathrm{CP}$. The Rosemont classification $(\mathrm{RC})$ is a method for diagnosing $\mathrm{CP}$ with the EUS criteria, which was introduced by a group of endosonography experts at an international consensus conference. In the RC, the ductal and parenchymal EUS findings are divided into major A, major B, and minor criteria. This RC classified the EUS findings as consistent with $\mathrm{CP}$, suggestive of $\mathrm{CP}$, indeterminate for $\mathrm{CP}$, or normal. ${ }^{6}$ Although the criteria are widely used at its current status, the diagnosis of CP using RC did not have high interobserver agreement.

The EUS strain elastography was reported as another diagnostic method for $\mathrm{CP}$, and it was shown to be correlated with

Correspondence to: Masayuki Kitano

Second Department of Internal Medicine, Wakayama Medical University, 811-1, Kimiidera, Wakayama 641-0012, Japan

Tel: +81-73-447-2300, Fax: +81-73-445-3616, E-mail: kitano@wakayama-med.ac.jp

Received on May 15, 2019. Revised on August 20, 2019. Accepted on September 12, 2019. Published online November 15, 2019. pISSN 1976-2283 eISSN 2005-1212 https://doi.org/10.5009/gnl19170

(c) This is an Open Access article distributed under the terms of the Creative Commons Attribution Non-Commercial License (http://creativecommons.org/licenses/by-nc/4.0) which permits unrestricted non-commercial use, distribution, and reproduction in any medium, provided the original work is properly cited. 
the CP stages of RC. ${ }^{8}$ However, the EUS strain elastography has some limitations. First, it cannot measure absolute value of hardness. Second, it is affected by the size and/or position of region of interest (ROI), because it is necessary to set the ROI in analysis. Different settings of ROI may cause heterogeneous results. On the other hand, EUS shear wave measurement (EUS$\mathrm{SWM}$ ) is a more precise modality for diagnosing $\mathrm{CP}$, because it provides absolute value of pancreatic hardness. However, there is no report of the usefulness of EUS-SWM, because it is newly introduced in the field of EUS. Therefore, the aim of this study was to evaluate the utility of EUS-SWM for diagnosing CP by comparing it with RC stages. We also sought to evaluate the utility of EUS-SWM for estimating CP severity by determining the endocrine dysfunction with presence of diabetes mellitus (DM).

\section{MATERIALS AND METHODS}

\section{Patients}

Between June 2018 and December 2018, 52 patients who underwent EUS and EUS-SWM were retrospectively enrolled in this study. The inclusion criteria were as follows: age $>20$ years; and EUS and EUS-SWM were performed on the same day; follow-up patients of CP or patients who were clinically suspected as $\mathrm{CP}$ with abdominal pain and/or history of recurrent pancreatitis. The exclusion criteria included malignant pancreatic lesion and previous pancreatic surgical procedure and/or total gastrectomy. EUS images were classified into four stages according to RC (consistent with $\mathrm{CP}$, suggestive of $\mathrm{CP}$, indeterminate of $\mathrm{CP}$, and normal). Subsequently, patients were classified into two groups: non-CP (including indeterminate of CP and normal) and CP (including consistent with $\mathrm{CP}$ and suggestive of $\mathrm{CP})$. Diagnostic concordance for CP between EUS and computed tomography (CT) was evaluated. This study was approved by the Ethics Committee of Wakayama Medical University (approval number: 2529).

\section{Study design}

This study was a retrospective study to evaluate the usefulness of EUS-SWM for CP. Primary outcome measurement was to evaluate the correlation between EUS-SWM and RC. The value of EUS-SWM was compared to RC stages and the number of EUS features in the RC. One of the secondary outcome measurements was to evaluate the diagnosis ability of EUS-SWM for $\mathrm{CP}$ and DM. The other secondary outcome measurement was to calculate the diagnostic ability of EUS-SWM and its cutoff point for diagnosing $\mathrm{CP}$, and to calculate the accuracy of EUS-SWM and its cutoff point for estimating CP severity by determining the endocrine dysfunction with presence of DM.

\section{EUS procedures}

Convex-type endoscopes (GF-UCT260; Olympus, Tokyo, Japan) with ultrasound (US) observation systems (ARIETTA 850; Hitachi Aloka Medical, Ltd, Tokyo, Japan) were used. EUS was performed in the left lateral position under diazepam-induced sedation with heart rate, saturation, and blood pressure monitoring. The endosonographer recorded EUS images for the later evaluation of RC. EUS-SWM was performed after recording the EUS images.

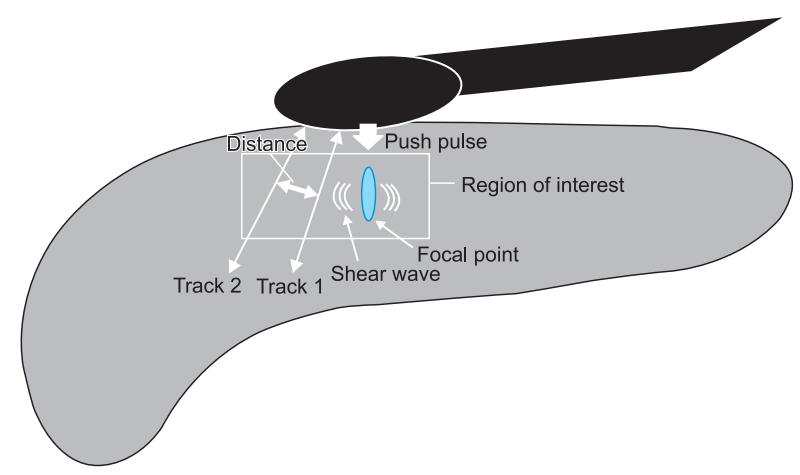

Fig. 1. Illustration of endoscopic ultrasonography shear-wave measurement.
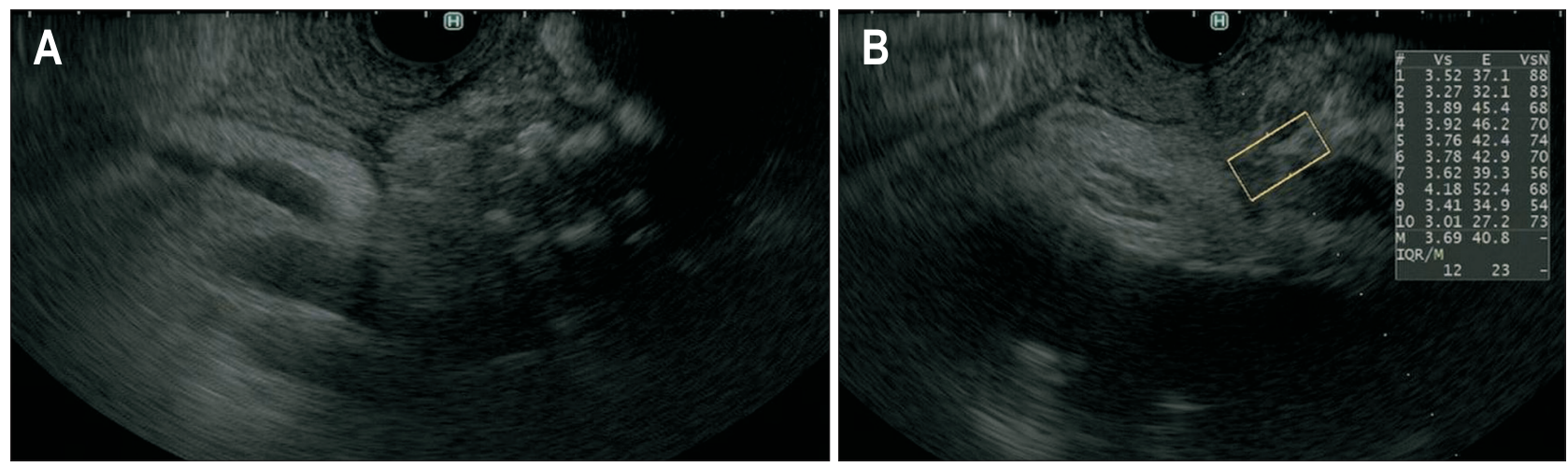

Fig. 2. Representative endoscopic images from a patient with chronic pancreatitis. (A) EUS showed a heterogeneous pancreas with strand, hyperechoic foci, and lobularity with honeycombing. (B) EUS-SWM was performed to diagnose chronic pancreatitis. The EUS-SWM value of 3.69 was higher than the cutoff value of 2.19 for diagnosing chronic pancreatitis.

EUS-SWM, endoscopic ultrasonography shear-wave measurement. 


\section{Shear wave measurement EUS elastography}

The detailed procedure and mechanism of action of EUSSWM are as follows: acoustic radiation force (push pulse) was sent to the focal point of the region of interest. Shear wave is generated at the edge by push pulse. The shear wave velocity (Vs; distance/ arrival time lag [m/s]) was calculated between two search points with track pulse (Fig. 1). If the tissue was hard, faster shear wave was propageted. The ROI $(5 \times 10 \mathrm{~mm})$ was set on the pancreatic parenchyma in the body without vessels, pancreatic duct, and cyst (Fig. 2). The value of EUS-SWM by shear Vs was measured ten times and evaluated with the median value. The percentage of the net amount of effective shear wave velocity (VsN, \%) showed which percentage of the measurement value was used in the calculation of Vs. The calculated VsN was used to assess whether or not the Vs value is reliable. In the previous reports, SWM-elastography with transabdominal US was calculated in three parts (head, body, and tail), and the body was shown to have the highest accuracy of diagnosing $\mathrm{CP}$ among the three positions. ${ }^{9}$ Therefore, we calculated the value of SWM-EUS in the pancreatic body.

\section{Rosemont classification}

Two endosonographers who were blinded to the results of EUS-SWM evaluated the recorded EUS images with reference to the RC. In the RC, ductal and parenchymal EUS findings were classified into major A, major B, and minor criteria. Moreover, this evaluation, based on the number and character of positive EUS criteria is classified as consistent with $\mathrm{CP}$, suggestive of $\mathrm{CP}$, indeterminate for $\mathrm{CP}$, or normal. When the three endosonographers had different conclusions, they re-evaluated together until an agreement was reached.

\section{Statistical analysis}

JMP Pro version 13 (SAS Institute Inc., Cary, NC, USA) was used for statistical analyses. The Fisher exact test for qualitative variables and Mann-Whitney $U$ test for quantitative variables, were used to compare categorical variables. The Dunnett test was used to compare the normal findings with each of the other findings (consistent with $\mathrm{CP}$, suggestive of $\mathrm{CP}$, and indeterminate of $\mathrm{CP}$ ). A difference was considered significant when the p-value was $<0.05$. The correlation between the value of EUSSWM and number of EUS features in the RC was performed with Spearman's correlation. The correlation coefficients $\left(r_{s}\right)$ were categorized as weak $(<0.2)$, mild $(0.2$ to $<0.4)$, moderate (0.4 to $<0.7$ ), or strong (>0.7) Diagnostic accuracy of EUS-SWM for $\mathrm{CP}$ was calculated after drawing the corresponding receiver operating characteristic (ROC) curve analysis. Based on the ROC curve analysis, a cutoff point was determined to maximize the Youden index. On this point, sensitivity and specificity were calculated. The area under the ROC (AUROC) accuracy was defined as low ( 0.5 to $<0.7)$, moderate $(0.7$ to $<0.9)$, or high $(\geq 0.9)$. The kappa coefficient was used for diagnostic concordance for $\mathrm{CP}$ between EUS and CT. Inter-rater agreement was assessed through magnitude and has been considered based on the Landis and Koch proposal as follows: Fleiss $\kappa$-values $<0$ as indicating no agreement; 0-0.20 as slight, $0.21-0.40$ as fair, 0.41-0.60 as moderate, $0.61-0.80$ as substantial, and $0.81-1$ as very good agreement. R3.4.1 (Integrated Development for R. RStudio, Inc., Boston, MA) was used for statistical analyses of inter-rater agreement.

\section{RESULTS}

In the 52 patients enrolled in this study, the mean age was $62.3 \pm 13.3$ years (range, 32 to 89 years). The RC diagnoses were consistent with $C P(n=10)$, suggestive of $C P(n=6)$, indeterminate of $\mathrm{CP}(\mathrm{n}=7)$, and normal $(\mathrm{n}=29)$. Accordingly, there were 36 and 16 patients in the non-CP and CP groups, respectively. Diagnostic concordance for $\mathrm{CP}$ between EUS and CT was very good ( $\kappa$-value, 0.83). Inter-rater agreement between non-CP and $\mathrm{CP}$ was very good agreement ( $\kappa$-value, 0.94). Demographic and clinical information of these patients is shown in Table 1. The CP group had higher proportion of male patients ( $\mathrm{p}=0.001)$, higher proportion of patients with DM ( $\mathrm{p}=0.026)$, and alcohol consumption (>80 g) $(\mathrm{p}=0.003)$ (Table 1).

Table 1. Characteristics of the Patients in the CP and Non-CP Groups

\begin{tabular}{|c|c|c|c|c|c|}
\hline \multirow[b]{2}{*}{ Variable } & \multicolumn{2}{|c|}{ Non-CP $(\mathrm{n}=36)$} & \multicolumn{2}{|c|}{$\mathrm{CP}(\mathrm{n}=16)$} & \multirow[b]{2}{*}{ p-value* } \\
\hline & $\begin{array}{c}\text { Normal } \\
(\mathrm{n}=29)\end{array}$ & $\begin{array}{l}\text { Indeterminate of CP } \\
\qquad(\mathrm{n}=7)\end{array}$ & $\begin{array}{l}\text { Suggestive of CP } \\
\qquad(\mathrm{n}=6)\end{array}$ & $\begin{array}{l}\text { Consistent with } \mathrm{CP} \\
\qquad(\mathrm{n}=10)\end{array}$ & \\
\hline Age, yr & $62.3 \pm 14.0$ & $63.0 \pm 13.4$ & $68.5 \pm 7.0$ & $58.2 \pm 14.8$ & 0.930 \\
\hline Sex, male/female & $7 / 22$ & $5 / 2$ & $6 / 0$ & $7 / 3$ & 0.001 \\
\hline BMI, $\mathrm{kg} / \mathrm{m}^{2}$ & $21.4 \pm 3.1$ & $22.0 \pm 3.0$ & $23.2 \pm 2.0$ & $23.2 \pm 2.5$ & 0.052 \\
\hline Diabetes mellitus & $3(10)$ & $1(14)$ & $1(16)$ & $5(50)$ & 0.026 \\
\hline Alcohol consumption (>80 g) & $1(3)$ & $2(29)$ & $4(67)$ & $3(30)$ & 0.003 \\
\hline
\end{tabular}

Data are presented as mean \pm SD or number $(\%)$. $\mathrm{CP}$, chronic pancreatitis; $\mathrm{BMI}$, body mass index. *Non-CP versus $\mathrm{CP}$. 
The median value of EUS-SWM for consistent with $\mathrm{CP}$, suggestive of $\mathrm{CP}$, indeterminate of $\mathrm{CP}$, and normal was 2.98 (2.29 to 4.52 ), 2.95 (2.19 to 3.76 ), 1.8 (1.24 to 3.02), and 1.52 (1.02 to 2.52), respectively. The value of EUS-SWM was significantly positively correlated with RC stages $\left(r_{s}=0.81\right)$, and the median value of EUS-SWM for consistent with $\mathrm{CP}$ and suggestive of $\mathrm{CP}$ were significantly higher than that for normal $(\mathrm{p}<0.001$ and $\mathrm{p}<0.001$, respectively) (Fig. 3). Moreover, the value of EUSSWM was significantly positively correlated with the number of EUS features in the RC ( $\left.\mathrm{r}_{\mathrm{s}}=0.72\right)$ (Fig. 4).

The AUROC for accuracy of EUS-SWM for diagnosing CP was 0.97 . By using the cutoff value of 2.19 , the sensitivity and specificity were 100\% and 94\%, respectively (Fig. 5).

On the other hand, for the accuracy of EUS-SWM for estimating $\mathrm{CP}$ severity by determining the endocrine dysfunction with presence of DM is present, the AUROC was 0.75. By using the cutoff value of 2.78 , the sensitivity and specificity were $70 \%$

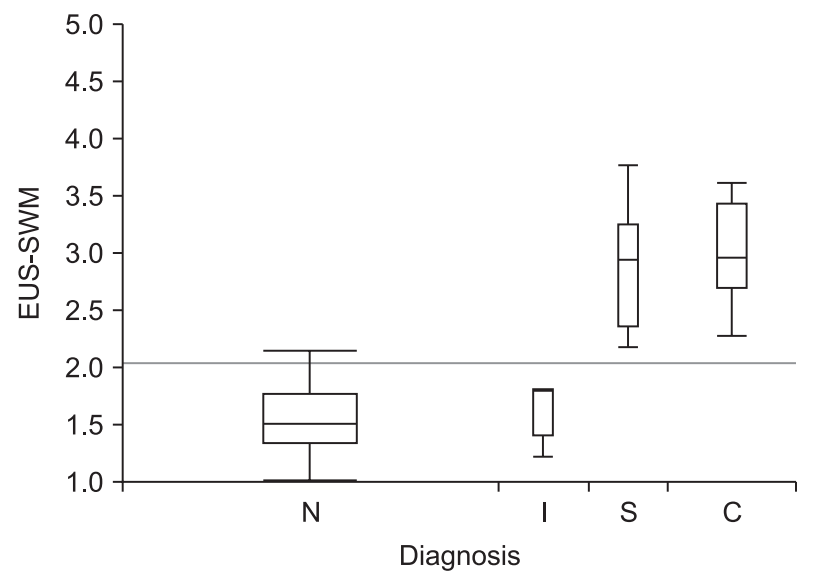

Fig. 3. Box and whisker plot of the value of endoscopic ultrasonography shear-wave measurement (EUS-SWM) for normal $(\mathrm{N})$ versus indeterminate of chronic pancreatitis (CP) (I), suggestive of CP (S), and consistent with $\mathrm{CP}(\mathrm{C})$ according to the Rosemont classification.

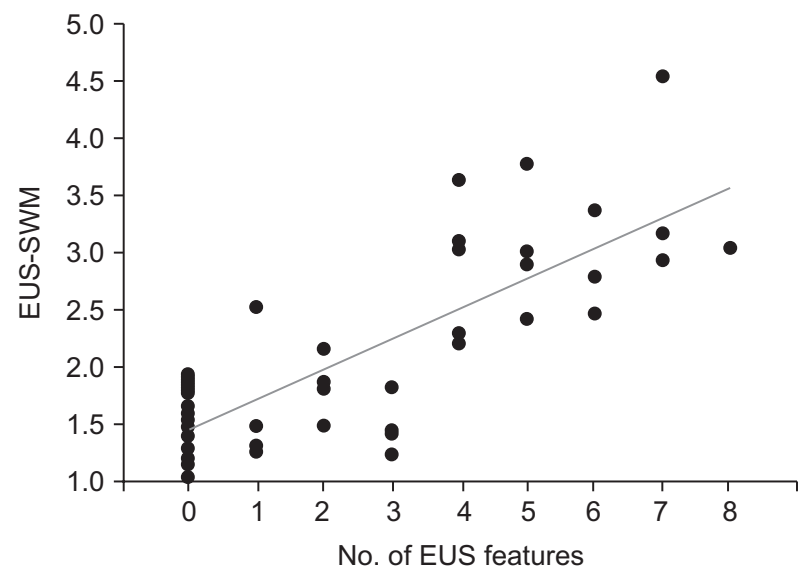

Fig. 4. Scatter plots of the value of shear wave measurement EUS elastography between the value of EUS-SWM and number of EUS features.

EUS-SWM, endoscopic ultrasonography shear-wave measurement. and 56\%, respectively (Fig. 6).

\section{DISCUSSION}

It is important to diagnose subclinical CP, because it may lead to exocrine and endocrine dysfunction, and pancreatic cancer in the future. Our study is the first report on the utility of EUSSWM for diagnosing CP. Nowadays, RC with EUS based on subjective judgement has been regarded as the most reliable diagnostic method for CP. However, this study demonstrated a very strong correlation between the absolute value of EUS-SWM and RC. Therefore, EUS-SWM can be an alternative diagnostic tool for $\mathrm{CP}$ based on objective judgement.

Although histology is the gold standard for the diagnosis of any disease, a histological diagnosis of CP can rarely be achieved because obtaining tissues routinely and safely is currently difficult. Therefore, we considered RC as the gold

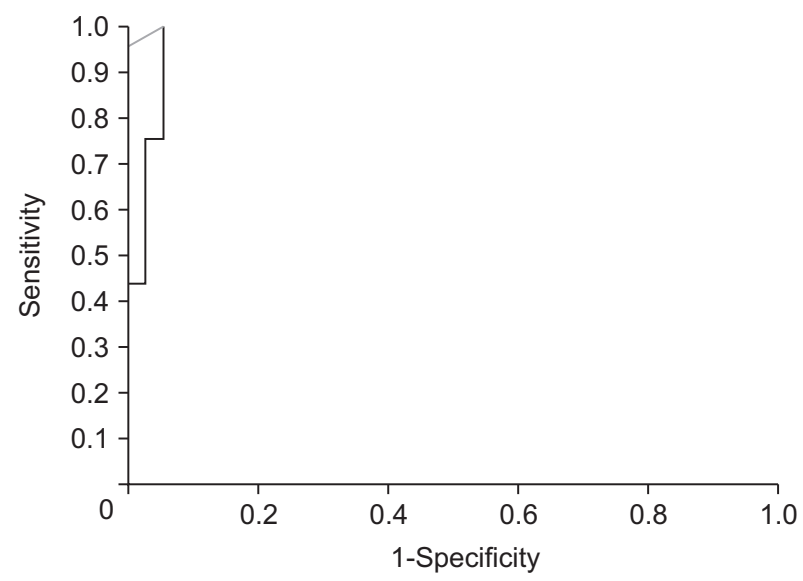

Fig. 5. The receiver operating characteristic curve for diagnosing chronic pancreatitis with endoscopic ultrasonography shear wave measurement.

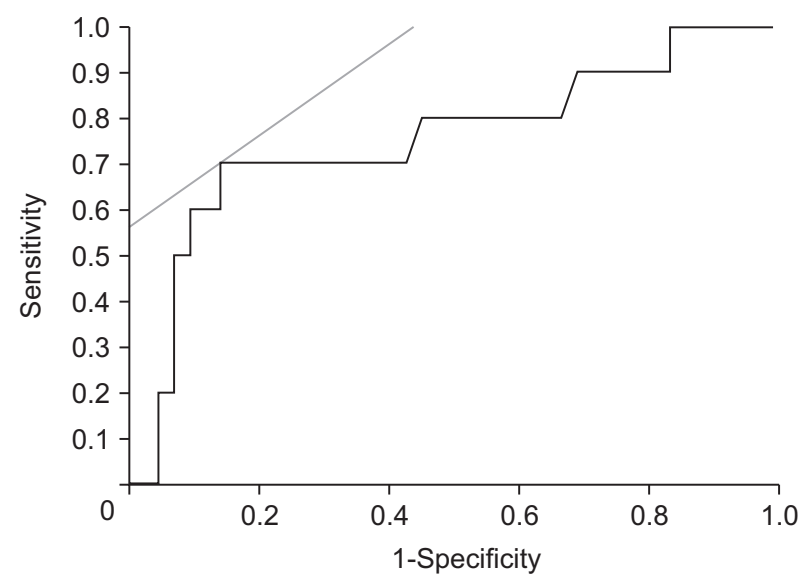

Fig. 6. The receiver operating characteristic curve for predicting diabetes mellitus with endoscopic ultrasonography shear-wave measurement. 
standard of diagnosing CP. Our study demonstrated that EUSSWM was significantly positively correlated with RC stages and the number of EUS features in the RC. In previous reports comparing EUS features and pancreatic fibrosis with histology, severity of fibrosis is closely correlated with the number of EUS features in the RC. ${ }^{10-12}$ Our results revealed that values of EUSSWM are closely correlated with the number of EUS features in the RC. Therefore, values obtained with EUS-SWM may reflect pancreatic fibrosis without performing histologic examinations. Moreover, measurement of absolute values in EUS-SWM can be more easily utilized for the assessment of time course of fibrotic changes and of the effect of treatment for CP compared to RC and conventional strain EUS elastography, because EUS-SWM is only a quantitative analysis method.

In terms of diagnostic ability of EUS-SWM for CP, the sensitivity, specificity, and AUROC were 100\%, 94\%, and 0.97, respectively. These data showed the excellent performance of EUS-SWM as a diagnostic tool for CP compared to other reports on elastography according to RC. Iglesias-Garcia et al. ${ }^{13}$ reported that the sensitivity, specificity, and AUROC curve of conventional strain EUS elastography for diagnosing CP were 91.2\%, 91\%, and 0.949, respectively. Considering our results, EUS-SWM seems superior to conventional strain EUS elastography. EUS-SWM has two advantages over conventional strain EUS elastography. First, EUS-SWM measures absolute value of hardness while conventional strain EUS does not. Second, conventional strain EUS elastography is affected by the size and/ or position of ROI because it is necessary to set ROI in different level of strain applied to the pancreas for analysis. On the other hands, EUS-SWM is a more precise modality for diagnosis of $\mathrm{CP}$ because it provides absolute value of pancreatic hardness.

SWM has already been reported in the field of transabdominal US. Kuwahara et al. ${ }^{14}$ reported that the sensitivity, specificity, and AUROC curve of transabdominal US-SWM for diagnosing CP were 77.1\%, 64.9\%, and 0.77, respectively. EUSSWM may have three advantages over elastography using transabdominal US. First, EUS overcomes the problems associated with US imaging caused by intervening gas, bone, and fat. Second, the image of pancreas is more clearly depicted in EUS than in transabdominal US because the EUS probe can be positioned closer to the pancreas unlike with transabdominal US. Third, it is difficult to set the ROI (10 mm) with transabdominal US because the width of the pancreas in $\mathrm{CP}$ is $<10 \mathrm{~mm}$ in some patients. In contrast, it is easier to set the ROI in the pancreas with EUS because the ROI of the EUS ( $5 \mathrm{~mm}$ ) is less than that of US (10 mm). Thus, EUS-SWM may seem to be a more excellent diagnostic method of $\mathrm{CP}$ than the other diagnostic methods for measuring elasticity of the pancreas.

$\mathrm{DM}$ is a common complication of $\mathrm{CP}$ and occurs naturally in $31.6 \%$ of $\mathrm{CP}$ patients. ${ }^{15}$ The micro- or macrovascular damage secondary to DM is a major threat in CP patients, affecting the mortality risk of these patients. ${ }^{16}$ In terms of the ability for pre- dicting the sensitivity, specificity, and AUROC of EUS-SWM for DM were 70\%, 56\%, and 0.75, respectively. The absolute cutoff values of EUS-SWM for diagnosing $\mathrm{CP}$ and for estimating its severity by determining the endocrine dysfunction with presence of DM were 2.19 and 2.78, respectively. Given that the cutoff value of EUS-SWM for estimating endocrine dysfunction is higher than that for diagnosing $\mathrm{CP}$, endocrine dysfunction may occur after advanced fibrosis in CP. In fact, duration from the onset of $\mathrm{CP}$ to the diagnosis of endocrine dysfunction is longer than the duration from the onset of $\mathrm{CP}$ to the diagnosis of $\mathrm{CP}{ }^{17}$ Therefore, EUS-SWM is also useful for estimating the severity of $\mathrm{CP}$, particularly in terms of endocrine dysfunction.

There are several limitations in this study. First, this study was a retrospective single-center study enrolling a small number of patients. Second, we employed RC as the gold standard, which is a subjective method for $\mathrm{CP}$ diagnosis. Further studies with a larger number of patients from multiple centers are required.

In conclusion, EUS-SWM provides objective assessment and can be an alternative tool for diagnosing CP. EUS-SWM may not only be useful for diagnosing $\mathrm{CP}$ but also for predicting endocrine dysfunction.

\section{CONFLICTS OF INTEREST}

The authors (M.K.) have received honoraria for speaking lectures in conferences from Olympus Corporation. The other authors declare that there are no conflicts of interest regarding the publication of this paper.

\section{ACKNOWLEDGEMENTS}

The present study was supported by grants from the Japan Society for Promotion of Science.

\section{AUTHOR CONTRIBUTIONS}

Drafting of the manuscript: Y.Y. Preparation for the manuscript: M.K. Data acquisition: Y.Y., Y.K., T.T., J.N., K.H., M. Itonaga, M.K. Analysis and interpretation of the data and critical revision of the manuscript for important intellectual content T.Y., Y.I., T.M., M. Iguchi. Statistical analysis: K.T.

\section{ORCID}

Yasunobu Yamashita

Kensuke Tanioka

Yuki Kawaji

Takashi Tamura

Junya Nuta

Keiichi Hatamaru

Masahiro Itonaga

Takeichi Yoshida https://orcid.org/0000-0002-6725-5708 https://orcid.org/0000-0001-6038-5844 https://orcid.org/0000-0001-8179-2641 https://orcid.org/0000-0002-5611-2509 https://orcid.org/0000-0002-3880-1546 https://orcid.org/0000-0001-9061-4361 https://orcid.org/0000-0002-0343-7246 https://orcid.org/0000-0003-0717-943X 
Yoshiyuki Ida

Takao Maekita

https://orcid.org/0000-0001-9698-9439

https://orcid.org/0000-0002-4160-7613

Mikitaka Iguchi

https://orcid.org/0000-0003-2579-2262

Masayuki Kitano

\section{REFERENCES}

1. Hirota M, Shimosegawa T, Masamune A, et al. The seventh nationwide epidemiological survey for chronic pancreatitis in Japan: clinical significance of smoking habit in Japanese patients. Pancreatology 2014;14:490-496.

2. Sze KC, Pirola RC, Apte MV, Wilson JS. Current options for the diagnosis of chronic pancreatitis. Expert Rev Mol Diagn 2014;14:199-215.

3. DeWitt J, McGreevy K, LeBlanc J, McHenry L, Cummings O, Sherman S. EUS-guided Trucut biopsy of suspected nonfocal chronic pancreatitis. Gastrointest Endosc 2005;62:76-84.

4. Ahmad NA, Kochman ML, Lewis JD, et al. Endosonography is superior to angiography in the preoperative assessment of vascular involvement among patients with pancreatic carcinoma. J Clin Gastroenterol 2001;32:54-58.

5. DeWitt J, Devereaux B, Chriswell M, et al. Comparison of endoscopic ultrasonography and multidetector computed tomography for detecting and staging pancreatic cancer. Ann Intern Med 2004;141:753-763.

6. Catalano MF, Sahai A, Levy M, et al. EUS-based criteria for the diagnosis of chronic pancreatitis: the Rosemont classification. Gastrointest Endosc 2009;69:1251-1261.

7. Stevens T, Lopez R, Adler DG, et al. Multicenter comparison of the interobserver agreement of standard EUS scoring and Rosemont classification scoring for diagnosis of chronic pancreatitis. Gastrointest Endosc 2010;71:519-526.

8. Kuwahara T, Hirooka Y, Kawashima H, et al. Quantitative diagnosis of chronic pancreatitis using EUS elastography. J Gastroenterol

2017;52:868-874.

9. Llamoza-Torres CJ, Fuentes-Pardo M, Álvarez-Higueras FJ, Alberca-de-Las-Parras F, Carballo-Álvarez F. Usefulness of percutaneous elastography by acoustic radiation force impulse for the noninvasive diagnosis of chronic pancreatitis. Rev Esp Enferm Dig 2016;108:450-456

10. Varadarajulu S, Eltoum I, Tamhane A, Eloubeidi MA. Histopathologic correlates of noncalcific chronic pancreatitis by EUS: a prospective tissue characterization study. Gastrointest Endosc 2007;66:501-509.

11. Chong AK, Hawes RH, Hoffman BJ, Adams DB, Lewin DN, Romagnuolo J. Diagnostic performance of EUS for chronic pancreatitis: a comparison with histopathology. Gastrointest Endosc 2007;65:808-814.

12. Albashir S, Bronner MP, Parsi MA, Walsh RM, Stevens T. Endoscopic ultrasound, secretin endoscopic pancreatic function test and histology: correlation in chronic pancreatitis. Am J Gastroenterol 2010;105:2498-2503.

13. Iglesias-Garcia J, Domínguez-Muñoz JE, Castiñeira-Alvariño $M$, Luaces-Regueira M, Lariño-Noia J. Quantitative elastography associated with endoscopic ultrasound for the diagnosis of chronic pancreatitis. Endoscopy 2013;45:781-788.

14. Kuwahara T, Hirooka Y, Kawashima H, et al. Usefulness of shear wave elastography as a quantitative diagnosis of chronic pancreatitis. J Gastroenterol Hepatol 2018;33:756-761.

15. Ryu JK, Lee JK, Kim YT, et al. Clinical features of chronic pancreatitis in Korea: a multicenter nationwide study. Digestion 2005;72:207-211.

16. Seicean A, Tantău M, Grigorescu M, Mocan T, Seicean R, Pop T. Mortality risk factors in chronic pancreatitis. J Gastrointestin Liver Dis 2006;15:21-26.

17. Pan J, Xin L, Wang D, et al. Risk factors for diabetes mellitus in chronic pancreatitis: a cohort of 2011 patients. Medicine (Baltimore) 2016;95:e3251. 